\title{
Era digital. Tendencias en la práctica educativa
}

\section{The digital age: trends in the practice of education}

DOI: https://doi.org/10.32870/dse.v0i22.729

\section{Gloria Yaneth Calderón Loeza*}

Pech Campos, S.; M. Prieto; J. García; E. Orozco (eds.). (2019). Innovation \& Practice in Education. Ciudad Real, España: Editorial CIATA.org

Desde la llegada de la era digital, la sociedad educativa se enfrenta a diversos desafíos en el paradigma de la enseñanza-aprendizaje (Pérez Gómez, 2013). Son solo aquellos comprometidos con la educación los que enfrentan y se adaptan a estos cambios, manteniendo la vanguardia en los avances educativos. Un ejemplo de ello es la Conferencia Conjunta Internacional sobre Tecnología y Aprendizaje (CCITA), dedicada a la innovación y la investigación en la educación, la cual se constituye a partir de una asociación entre investigadores educativos de diversas partes del mundo, principalmente Estados Unidos e Iberoamérica, cuyos objetivos son congregar, establecer redes, publicar y divulgar los nuevos conocimientos expuestos en las conferencias anuales desde el año 2009 (Ciata.org, 2020).

Referente a estos objetivos, en su última reunión realizada en el año 2019, la CCITA presentó el undécimo libro, titulado Innovation \& Practice in Education, el cual reúne las contribuciones de algunos conferencistas del evento. La obra está compuesta por 41 artículos de investigación afines a la innovación educativa y tecnológica, con un predominio del idioma español. Está editado por Silvia J. Pech Campos, Manuel E. Prieto, Javier García y Eduardo Orozco, quienes pertenecen a la CIATA, y por otros más de 100 miembros del comité científico, caracterizados por ser investigadores con el máximo grado académico en su área, pertenecen a universidades de prestigio y tienen experiencia en tecnologías. Entre los países participantes se encuentran Estados Unidos, México, Argentina, Brasil, Uruguay, Perú y España.

El contenido del libro se divide en tres secciones. La primera, "Innovación educativa. Nuevos aportes", está compuesta por 24 capítulos que abordan temáticas relacionadas con los métodos de enseñanza, la aceptación de los recursos tecnológicos y las necesidades formativas de profesores y alumnos. Leyva Carrillo, Carreño León, Sandoval Bringas, Estrada y Espinoza Monteverde presentan un estudio realizado en México, en donde la modalidad M-learning (aprendizaje

* Alumna de la Maestría en Investigación Educativa. Licenciatura en Medicina General. Líneas de investigación: currículum, tecnologías educativas, educación en salud. Universidad Autónoma de Yucatán. México. egloriacalderon@correo.uady.mx 
electrónico móvil) se encuentra en fase de implementación. Se aplicó un método cuantitativo con recogida de datos mediante encuesta, el cual arrojó resultados favorables para la modalidad mixta, lo que determina la aceptación de los dispositivos móviles como herramientas de aprendizaje y comunicación entre docentes y alumnos.

Por su parte, Domínguez, May Ayuso, Méndez Ojeda, Zapata González y Hernández asociaron el rendimiento académico con el uso del smartphone en un grupo de estudiantes de bachillerato; mediante un cuestionario de medición tipo Likert obtuvieron resultados favorables en el uso del teléfono celular como herramienta educativa. May Ayuso, Suárez Baeza, Alpuche Rivera y Quiñonez-Pech presentaron un estudio con metodología cualitativa en Yucatán (México), el cual, mediante entrevistas a los formadores del Programa de Inclusión y Alfabetización Digital (PIAD), se describió la percepción del profesorado ante la integración de la tableta digital como herramienta tecnológica en el proceso de la enseñanza-aprendizaje. Los resultados arrojaron que los profesores presentan dificultades en el manejo de los grupos, la motivación y la brecha digital, aunque estos dos últimos lograron minimizarse tras la implementación del PIAD.

Los tres estudios citados evidencian cómo la era digital ha marcado una brecha generacional entre docentes y alumnos, que influye en las formas de comunicar, enseñar y aprender. Los alumnos actuales, a los que Prensky (2010) denomina nativos digitales, tienen una facilidad innata de buscar y encontrar información a través de los recursos tecnológicos; con el internet como fuente de conocimiento inagotable, el nativo digital presenta características que lo diferencian de sus antecesores (profesores) pues prefiere recibir la información de forma ágil e inmediata, se siente atraído por las multitareas y rinde mejor cuando trabaja en red. Esto concuerda con los resultados obtenidos por López Sánchez, González Romero, López Sotelo y Rocha Ruiz, quienes encontraron que las competencias digitales de los estudiantes se encuentran en el nivel de exigencia que requiere la universidad; sin embargo, los alumnos reportan que dichas competencias las adquieren de forma autónoma, es decir, sin la instrucción de las instituciones educativas.

Además de la percepción concebida por el alumno, sería de utilidad evaluar la parte práctica de las competencias digitales y si corresponden o no al nivel referido. Otro aspecto para considerar sería confirmar si las instituciones educativas, en conjunto con el personal administrativo y el profesorado, cumplen con el grado de alfabetización digital necesaria para poder trasmitir de forma satisfactoria el nivel de competencia digital requerida por sus alumnos. En este estudio vuelven a hacerse notar las necesidades formativas de los profesores.

En Uruguay, Albana Sanz resalta la preocupación por el estado emocional del alumno al presentar una intervención educativa con previa capacitación del profesorado, la cual fomenta la mejora del clima escolar y previene la violencia. Esta propuesta es similar a la del Programa Nacional de Convivencia Escolar (PNCE) en México, que promueve la prevención, formación y convivencia escolar, por lo que podría ser útil para su abordaje investigativo.

Diólo@os sobre Educación TEMAS ACTUALES EN INVESTIGACION : año 12 | número 23 | julio-diciembre 2021 | ISSN 2007-2171 
La segunda sección, "Innovación tecnológica. Nuevos aportes", está compuesta por 12 artículos relacionados principalmente con el uso de herramientas virtuales y aplicaciones móviles en los procesos de enseñanza-aprendizaje. Entre las temáticas más relevantes se encuentra la promoción de la educación ambiental en los alumnos de educación básica mediante videojuegos, propuesta por Sandoval Bringas, Carreño León, Leyva Carrillo, Estrada Cota, Sandoval Carreño. Aunque podría resultar paradójica la utilización de un recurso artificial para producir una conciencia ambiental real, se constata su aceptación mediante una metodología cuantitativa basada en encuestas.

Por otro lado, Olivares Carmona, Ortiz Pacheco, Angulo Armenta y Olivares Carmona, a través de un estudio de caso, produjeron material tiflotécnico para apoyar las necesidades inclusivas y de aprendizaje de un estudiante con discapacidad visual total. Se obtuvieron 42 recursos educativos en formato de audio, los cuales fueron proporcionados al participante a través de correo electrónico y WhatsApp. De esta manera se obtienen recursos reproducibles y permanentes para este grupo vulnerable de la sociedad, promoviendo la inclusión y la permanencia académica.

La tercera sección, "Proyectos en desarrollo", está compuesta por cinco capítulos que hacen referencia al desarrollo humano y sustentable en el proceso de enseñanza-aprendizaje. Espinosa López, Torres Gastelu y Pech Campos dan a conocer los cuidados y los malos hábitos respecto a la privacidad y seguridad digital en estudiantes universitarios, manifestando ser conscientes de los peligros a los que se expone su información en la red y la falta de cuidado al proteger contraseñas u omitir la lectura de las políticas de páginas web. Estos resultados sugieren la necesidad de orientación para el alumno respecto al manejo de sus datos personales en las redes.

Como respuesta a esta problemática, Prats, Torres Rodríguez, Oberst y Carbonell (2018) expusieron un estudio basado en un diseño y aplicación de talleres educativos para el uso saludable de internet y redes sociales en la adolescencia, el cual reporta resultados favorables en su aplicación al minimizar los efectos negativos derivados de las Tecnologías de Información y Comunicación (TIC). Como recurso de apoyo, proporcionan una guía de recomendaciones para la vigilancia y seguridad digital para que los padres y maestros apliquen a lo largo del desarrollo y crecimiento del estudiante.

De manera general, el libro en cuestión, en su diversidad temática nos expone un panorama amplio de escenarios y contextos sociales nacionales e internacionales en el que se incluye a profesores y alumnos de niveles básicos, universitarios, con necesidades especiales o en estado de vulnerabilidad, y en los que la innovación educativa, tecnológica y de desarrollo son trascendentales en los procesos de enseñanza-aprendizaje. Se resalta la preparación del profesorado como punto clave en la mejora del sistema educativo. En mi opinión, considero que algunos estudios presentan limitaciones que no se describen en el texto, produciendo un sesgo en su interpretación para quienes no tienen conocimiento en estadística e investigación. Además de 
carecer de literatura científica que los sustente, algunos solo cuentan con cuatro o cinco referencias bibliográficas. Otro aspecto para considerar son los métodos estadísticos empleados que, a mi parecer, deben emprender nuevas rutas ya que la mayoría de los estudios realizados permanecen en percepciones individuales y grupales. Esto no quiere decir que sean inválidos o refutables, pero sería enriquecedor leer mayor diversidad en su abordaje como, por ejemplo, considerar métodos multivariados y métodos mixtos (cuantitativos y cualitativos) que consideren la realidad compleja del paradigma actual en los procesos de enseñanza-aprendizaje.

El libro Innovation \& Practice in Education está dirigido a investigadores, administradores, maestros y alumnos interesados en mantenerse a la vanguardia de los conocimientos educativos en la era digital, promueve la discusión sobre los métodos de estudio empleados y brinda información sobre las necesidades de programas y prácticas con el fin de mejorar la calidad educativa.

\section{Referencias}

Ciata.org. (2020). La Asociación. 22/03/2020, de Organización de la Conferencia Conjunta Iberoamericana sobre Tecnologías para el Aprendizaje.

https://sites.google.com/view/ciataorg/ciata-org/la-asociación-ciata-org

Prats, M.; A. Torres Rodríguez; U. Oberst; X. Carbonell (2018). Diseño y aplicación de talleres educativos para el uso saludable de internet y redes sociales en la adolescencia: Descripción de un estudio piloto. Revista de Medios y Educación, 115.

http://dx.doi.org/10.12795/pixelbit.2018.i52.08

Prensky, M. (2010). Nativos e inmigrantes digitales. Cuadernos Sek 2.0.

http://marcprensky.com/writing/Prensky-NATIVOS E INMIGRANTES DIGITALES (SEK).pdf 